\title{
Assessment of knowledge, attitude and practice of insulin injection among subjects with diabetes mellitus
}

\author{
Bezawada Shravani ${ }^{1 *}$, Anuradha H. V. ${ }^{1}$, Pramila Kalra ${ }^{2}$
}

Department of ${ }^{1}$ Pharmacology, ${ }^{2}$ Endocrinology, Ramaiah Medical College, Bengaluru, Karnataka, India

\author{
Received: 02 July 2021 \\ Revised: 02 August 2021 \\ Accepted: 03 August 2021 \\ *Correspondence: \\ Dr. Bezawada Shravani, \\ Email: shravani921@gmail.com
}

Copyright: $@$ the author(s), publisher and licensee Medip Academy. This is an open-access article distributed under the terms of the Creative Commons Attribution Non-Commercial License, which permits unrestricted non-commercial use, distribution, and reproduction in any medium, provided the original work is properly cited.

\begin{abstract}
Background: Diabetes mellitus (DM) is a spectrum of common metabolic disorders whose management mainly lies in treating the patients with oral hypoglycemic drugs and insulin along with the dietary and lifestyle modifications. Insulin is administered most subcutaneously. As the insulin injection sites are relatively painless, patients tend to inject in the same area repeatedly rather than moving to a newer site and increase risk for development of injection site reactions like lipodystrophy and impairment of glycemic control. Hence, it is utmost important for every diabetic patient and their relatives who would inject the insulin injection to be aware of appropriate manner of insulin injection. This helps in maintaining adequate glycemic control in diabetic patients. The main objective of this study was to assess the knowledge, attitude and practice of insulin injection technique among the diabetic patients.

Methods: A cross-sectional study was conducted in the department of Endocrinology on 250 diabetic patients taking insulin injections based on inclusion and exclusion criteria. A validated questionnaire was administered to patients to answer in order to assess their knowledge, attitude and practice about technique of insulin injection.

Results: In this study, it was found that $90 \%$ of the patients were aware of rotating the injection site, whereas only $40 \%$ of the patients were aware of appropriate time duration (5-10 sec for syringes/counting 1-15 for releasing the pen) required for resting the syringe/pen needle inside the skin. Only $48 \%$ of the patients used to remove the air bubble prior to injection, 57\% pinched the injection site before injecting, 20\% rubbed the injection site after injection and $30 \%$ used to wash hands prior to injection. Hypoglycemia was the most common adverse effect noted in $54 \%$ of patients.

Conclusions: It can be concluded from the present study that every patient and his/her attendant needs to be educated and trained appropriately regarding technique of injecting insulin injection for betterment of their health.
\end{abstract}

Keywords: Insulin, Knowledge, Attitude, Practice of insulin injection

\section{INTRODUCTION}

Diabetes mellitus (DM) is a spectrum of common metabolic disorders caused due to genetic and environmental factors involving insufficient secretion of insulin, reduced responsiveness to endogenous or exogenous insulin. ${ }^{1}$ With the technological advances there has been increase in the number of patients diagnosed with DM and treated with insulin in initiastages. ${ }^{2}$ Insulin is the drug of choice in type 1 diabetes and also for treating type
2 diabetes along with other anti-diabetic medications. ${ }^{1}$ It is most commonly administered via subcutaneous route. ${ }^{3}$

Insulin therapy can lead to development of adverse drug reactions at injection site like lipohypertrophy/lipoatrophy, bruising and allergic reactions etc., which can further lead to development of poor metabolic control and glycemic variability. ${ }^{4}$ Multiple factors play role involving the technique of insulin injection leading to such adverse drug reaction. $^{2}$ Hence, patients should be taught to examine their own injection sites and educated regarding 
appropriate technique of injection and manner of rotating injection sites for early recognition of any skin changes. They should be motivated to undergo periodic health check-up along with practicing correct injecting techniques for better health care as Insulin once started needs to be taken for lifelong based on glucose levels. ${ }^{2,4}$

There is a need to understand and know the knowledge, attitude and practice of insulin injecting techniques by diabetic patients, so that adequate education could be provided to the patients regarding insulin injection techniques along with awareness of insulin related adverse drug reactions which could improve the healthcare quality of life.

\section{Objective}

Objectives of the study were to assess the knowledge, attitude and practice of insulin injection techniques among the diabetic patients.

\section{METHODS}

A cross-sectional study was conducted among the 250 diabetic patients who visited the department of endocrinology at M. S. Ramaiah medical college and hospital from December 2015 to March 2017 for a period of one year three months. The study was ethically approved by institutional ethics committee prior to commencement. A written informed consent was taken from all the patients who were willing to participate in the study. A series of questions were presented to diabetic patients (either on an outpatient consultation or inpatients) under the department of endocrinology. Post assessing the eligibility criteria, these patients were provided with set of questionnaires designed pertaining to technique of insulin injection. Thereafter, the responses to the questionnaire were assessed along with interpretation of results. Statistical analysis-all the quantitative variables like age, duration of DM, BMI etc., were presented using descriptive statistics like mean, median, range and standard deviation. All the qualitative variables like frequency of insulin injections, gender etc., were presented using frequencies and percentages. The participants and their relatives who followed incorrect methodology were further counselled about the correct technique of insulin injection.

\section{Inclusion criteria}

Diabetic patients-type 1 or type 2 , aged 18 years and above, on insulin therapy and willing to give informed consent were included in the study.

\section{Exclusion criteria}

Pregnant and lactating mothers were excluded from the study.

\section{RESULTS}

In this study, a total number of 250 diabetic patients on insulin therapy were included. Table 1 summarizes age and gender distribution with various other characteristics of diabetic patients in this study, which showed there is slightly male preponderance and majority of patients were adults with a mean age of 55 years. Table 2 summarizes the percentage of various aspects of knowledge attitude practice of insulin administration. Among $86 \%$ of patients who stored insulin vial or cartridges in fridge; $84 \%$ stored in fridge whereas, the rest $2 \%$ in earthen pots. Those who used fridge for storage used appropriately the lower compartment of it and not in the freezer. Rubbing at the injection site after administration of the insulin was done by $20 \%$. Rubbing can cause localized minute hemorrhages or bruises. Three patients with lipodystrophy presented with bruise and mild hemorrhage at the injection site. Approximately $57 \%$ of the diabetic patients pinched the site of injection/raise the skin to inject the insulin. Majority of the patients used to inject insulin $15-20 \mathrm{~min}$ prior to consumption of food however 2 of them used to take insulin after eating meals. The most commonly used insulin syringes were of $40 \mathrm{cc}$ followed by $100 \mathrm{cc}$ with most common needle size 6-or 8-mm. Insulin pens injected by these patients were mostly $4 \mathrm{~mm}$ in size. In this study, diabetic patients on insulin therapy used to change needle of insulin pens and syringes once in 3-4 days and most commonly after injecting 6/more injections (Table 2).

Table 1: Demographic data of diabetic patients on insulin therapy.

\begin{tabular}{|l|l|}
\hline Variables & Number of patients (\%) \\
\hline Females & $108(43)$ \\
\hline Males & $142(57)$ \\
\hline Age range (Years) & $18-89$ \\
\hline Mean age (Years) & $55 \pm 13.8$ \\
\hline History of smoking & $65(26)$ \\
\hline History of alcohol intake & $70(28)$ \\
\hline Type of diabetes & Type 1-5 patients \\
\hline Family history of DM & Type 2-245 patients \\
\hline Mean duration of DM (Years) & $69(28)$ \\
\hline
\end{tabular}


Table 2: Knowledge, attitude and practice of insulin injection among subjects.

\begin{tabular}{|l|l|}
\hline Questionnaire pertinent to insulin injection techniques & Percentage (\%) \\
\hline Hypoglycemia & 54 \\
\hline Storage of insulin vials/cartridges (fridge/earthen pot) & 86 \\
\hline Expiry date not checked & 49 \\
\hline Rotation of site of injection & 90 \\
\hline Change of insulin pen needles and syringes & Once in 3-4 days or after 6 injections \\
\hline Removal of air bubble from syringe prior to injection & 48 \\
\hline Hand wash prior to injection & 30 \\
\hline Rubbing the site after injection & 20 \\
\hline $\begin{array}{l}\text { Awareness about resting the syringe needle inside skin for 5-10 } \\
\text { sec/counting 1-15 number then lifting the pen from skin }\end{array}$ & 40 \\
\hline Pinching the site prior to injection & 57 \\
\hline Insulin +oral hypoglycemic agents & 66.8 \\
\hline Awareness with respect to types of DM & 40 \\
\hline Syringes most commonly used (CC) & 40 or 100 \\
\hline Needle length of syringes (mm) & 6 or 8 \\
\hline Needle length of insulin pens (mm) & 4 \\
\hline
\end{tabular}

\section{DISCUSSION}

It is utmost important for every diabetic patient and their relatives who would inject the insulin injection to be aware of appropriate manner of insulin injection. In this study we tried to understand the knowledge, attitude and practice of insulin injection technique. Correct guidelines need to be followed in order to reduce the incidence of lipodystrophy due to insulin injection. This helps in maintaining adequate glycemic control in diabetic patients. Therefore, it is important to educate diabetic patients and their attenders with respect to appropriate technique of insulin injection. There is male preponderance compared to female in the study and with an average age group of 55 years. In accordance with recent guidelines for insulin injection technique forum for injection technique (FIT) Ireland, 2012 and studies conducted by Mary et al, Samaira et al and Bahar Vardar et al, a series of questions were compiled and validated..$^{5-8}$ After the questionnaire was answered, patients and their relatives were counselled about the appropriate technique of insulin injection personally. Those patients who were found to be practicing incorrect technique of administration of injections were recounselled regarding appropriate technique of injection.

In a similar study conducted by Ramalakshmi et al, in diabetic centre of Lagos state university teaching hospital, the adverse effects of insulin and hypoglycaemic therapy was assessed with the help of questionnaire consisting of nine questions assessing the knowledge of insulin, storage requirements of insulin, site change during insulin therapy, discomfort during therapy, dosage adjustments, dietary modifications, management of hypoglycaemic conditions and complications of diabetes. ${ }^{9}$ It was observed that patient's knowledge about their insulin preparation and dosage increased when they were counselled for the same and also the incidence of adverse effects came down significantly with proper counselling of the patients. ${ }^{10}$
Along with insulin injection, $66.8 \%$ used to consume oral hypoglycaemic agents and hypoglycaemia was experienced in $54 \%$ of these patients (Table 2). Weakness and dizziness were the most common symptoms. In a survey conducted by Shriraam et al, among 2530 Americans with type 2 diabetes $55 \%$ reported having experienced hypoglycaemia. ${ }^{11}$ In another retrospective, an interview-based study by Miller et al, among 1055 type 2 diabetics, the prevalence of hypoglycaemia was $16 \%$ in those consuming OHAs and in $30 \%$ of those injecting insulin. ${ }^{12}$ Glucose (15-20 gm) is the preferred treatment for the conscious individual with hypoglycemia, although any form of carbohydrate that contains glucose may be used. The individual should consume a meal or snack to prevent recurrence of hypoglycaemia. ${ }^{11}$ Also, based on the recommendations by American diabetic association (ADA) and forum for injection of insulin (FIT) the results of this study were compared in order to understand how insulin injection needs to be appropriately administered in order to reduce the frequency of insulin induced related adverse effects. ${ }^{13,14}$ Limitation of this study is lack of follow up of all patients after counselling for following the appropriate technique as this study was mainly cross-sectional.

\section{CONCLUSION}

The questionnaire which was administered to assess the knowledge, attitude and practice of insulin injection determined that majority of the patients were rotating injection sites and storing insulin vials/cartridges appropriately. However, checking expiry dates of insulin vials/cartridge, frequency of changing needles/syringes, hand washing and pinching the skin etc., still needs to be practiced efficiently. Those patients who were found to be practicing incorrect technique of administration of injections or those who were unaware of appropriate technique were re-counselled with respect to correct 
method of insulin administration. The observations of the present study have generated information about prevalence of insulin induced lipodystrophy along with knowledge, attitude, and practice of injection technique. Hence it can be concluded that active surveillance of adverse reaction like insulin induced lipodystrophy should be done as it is preventable, which further could help in avoiding variability in glycemic levels among diabetic patients who are on long term insulin therapy. Diabetic patients and their relatives should be well educated with respect to appropriate technique of insulin.

Funding: No funding sources

Conflict of interest: None declared

Ethical approval: The study was approved by the Institutional Ethics Committee

\section{REFERENCES}

1. American Diabetes Association. Diagnosis and Classification of Diabetes Mellitus. Diabetes Care. 2012;35(1):64S-71.

2. Kadiyala $\mathrm{P}$, Walton $\mathrm{S}$, Sathyapalan $\mathrm{T}$. Insulin Induced Lipodystrophy. Bri J Diabetes Vascular Dis. 2014;14(4):131-3.

3. Kansra UC, Sircar. Insulin Therapy: Practical Points. J Indian Academy Clin Med. 2000;1(3):245-51.

4. Ajlouni MA, Abujbara M, Batieha A, Ajlouni K. Prevalence of Lipohypertrophy and associated risk factors in Insulin-treated patients with Type 2 Diabetes Mellitus. Int $\mathbf{J}$ Endocrin Metab. 2015;13(2):e20776.

5. Gentile S, Strollo F, Ceriello A. Lipodystrophy in Insulin-Treated Subjects and Other Injection-Site Skin Reactions: Are We Sure Everything is clear? Diabetes Therapy. 2016;7(3):401-9.

6. Mary T, Malachi Mc K. Lipohypertrophy In Insulin-Treated Diabetes: Prevalence and Associated Risk Factors. J Diabetic Nursing.
2015;17:340-43.

7. Samaira E, Utoor J, Doaa B. A study to access the attitude and practice of diabetic patient towards selfadministration of insulin in Basra city, Iraq. Int $\mathrm{j}$ med pharmacy. 2014;3(4):65-74.

8. Vardar B, Sevgi K. Incidence of lipohypertrophy in diabetic patients and a study of influencing factors. Diabetes Res Clin Practice. 2007;77(2):231-6.

9. Ramalakshmi S, Madhuri S, Kousalya K, Saranya P. A study on insulin usage among diabetic patient in a tertiary care teaching hospital. Indo Am J pharmaceutical res. 2013;3(10):8474-9.

10. Frid AH, Hirsch LJ, Menchoir AR, Morel D, Strauss $\mathrm{K}$. Worldwide injection technique questionnaire study: population parameters and injection practices. Mayo Clinic Proc. 2016;91(9):1212-3.

11. Shriraam V, Mahadevan S, Anitharani M, Jagadeesh $\mathrm{N}$, Kurup S, Vidya T. Reported hypoglycemia in Type 2 diabetes mellitus patients: Prevalence and practices-a hospital- based study. Indian J Endocrinol Metabolism. 2017;21(1):148-53.

12. Miller C, Phillips L, Ziemer D, Gallina D, Cook C, El-Kebbi I. Hypoglycemia in Patients with Type 2 Diabetes Mellitus. Arch Internal Med. 2001;161(13):1653-9.

13. American Diabetes Association. Insulin Administration. Diabetes Care. 2004;27(1):S106-9.

14. FIT Ireland. The First Irish Injection Technique Recommendations. $1^{\text {st }}$ ed. 2012. Available at: http://fit4diabetes.com/files/9413/5876/6815/FITRec ommendationsIreland.pdf. Accessed on 18 August 2017.

Cite this article as: Shravani $\mathrm{B}$, Anuradha HV, Kalra P. Assessment of knowledge, attitude and practice of insulin injection among subjects with diabetes mellitus. Int J Basic Clin Pharmacol 2021;10:1130-3. 